\title{
Towards Policy Decomposition for Autonomic Systems Governance by Applying Biologically Inspired Techniques.
}

\author{
John Keeney, Vincent Wade \\ Knowledge \& Data Engineering Group, School of Computer Science and Statistics, \\ Trinity College Dublin, Dublin 2, Ireland. \\ \{ John.Keeney | Vincent.Wade \}@cs.tcd.ie
}

\begin{abstract}
Policy-based management systems use declarative rules to govern their operation whilst satisfying the goals of the system. One of the fundamental issues in policy engineering remains the inability to automatically refine high-level goals to low level achievable goals, many of which will be interdependent and conflicting. This paper introduces the automated decomposition and refinement of management policies through the optimisation of balancing constraints by the innovative integration metaheuristic algorithms.
\end{abstract}

\section{Introduction}

As networks, distributed computing and pervasive computing converge, an increasing trend can be observed for making elements in a network more autonomous and easy to manage. An autonomic system is an adaptive computer system that self-manages, driven by high level governance directives rather than low level management policies [1]. This governance is performed through the definition of goals and constraints for the systems, i.e. defining 'what' the system should be doing, rather than explicitly stating 'how' the system should achieve its goal. In the autonomic systems considered in this paper these governance directives would come from a human overseeing the overall operation of the system. These humans, which we term Governors, should not need to know the intricacies of each part of the autonomic system to be able to govern it and affect the overall behaviour of the system. The goal of autonomic management is to reduce the cognitive load on the manager and reduce the cost of ownership of the managed system, especially as the management of the system more costly and more complex over time. This is particularly obvious in highly adaptive services across heterogeneous networks to support diverse applications and devices.

However, a key problem in autonomic management remains the lack of a structured mechanism to determine what is meant by high-level goal concepts used in governance directives, and how these high level objectives can be decomposed into low level objectives that can be measured, constrained and enforced. This is particularly difficult if the individual objectives that make up a high level goal are constrained or conflicting [2] in some way and need to traded-off against each other to achieve multidimensional balancing of objectives to meet that goal. This paper focuses on how these decomposed multiple objectives can be globally optimised to achieve the governor's goal.

\section{Policy Decomposition and Refinement}

Policy-based management uses explicit declarative rules to govern the adaptation of managed systems, whilst satisfying the goals of the system. Policy-based management began as a technique to simplify the management of network devices into sets of rules that together would maintain correct operation of a network. Quickly, these rule bases became too complicated to be manipulated, resulting in the need to define higher level rules or "policies" to constrain the operation of the lower level rules. Policy refinement is the process of transforming a high-level abstract policy specification into low-level concrete ones that can be enforced to achieve that policy. Therefore a policy refinement technique must link high level goals with underlying system metrics and adaptabilities in order to derive the different ways in which the system can satisfy the goal [3]. As far back as 1993 experts in policy based management identified the decomposition of such high-level goals to application level goals as an operation that could only be performed by a human or possibly by some domain-specific expert system [4][5]. To date an automated domain-independent goal decomposition mechanism has not emerged. A case-based reasoning approach is proposed in [6] to assist the refinement process based on the clustering of goals in previous refinements, but requires a large training set of previous refinement operations. A semi-manual approach 
for addictively reasoning over available sub-goals into super-goals is suggested in [3], but this system only operates on previously refined sub-goals. Other work in [7][8] focuses on taking low level policies and iteratively composing and abstracting them to present these higher level policies as possible governance policies, which can then be decomposed back into lower level policies. This approach builds a policy hierarchy from the bottom up as opposed to the top-down approach advocated in [5]. While this two-way approach seems to hold most promise as general purpose tool for policy decomposition, the major problem lies in the combination of conflicting lower-level policies into a single combined policy for use at a higher level. The key challenge discussed in this paper remains the lack of a general-purpose automated way to explicitly capture how sub-policies can be combined in a constrained and balanced way to achieve the Governor's goal.

\section{Multi-Objective Optimisation}

This research is primarily focused on optimisation and combination of multiple conflicting objectives to achieve an optimum strategy to meet a Governor's goal. Multiobjective constraint optimisation has its roots in the mid20th century micro-economics of game theory where the choice of conflicting strategies was driven by the optimisation of conflicting utility for all players. Much of the research on optimising conflicting objectives focuses on identifying a utility or fitness function to provide a numerical representation of preference ordering of choices. This function would then be solved using multi-order differential algebra to achieve a globally optimal balance between inputs. While this method works well for equations with a small set of inputs with clearly defined mathematical relationships between each, it fails where higher order differential equations become too difficult to solve, or there does not exist a solvable deterministic relationship between all inputs. Since many of these Multi Objective Optimisation (MOO) problems cannot be solved numerically, a heuristic approach is required.

More recent research in biologically inspired metaheuristic artificial intelligence and evolutionary algorithms focuses on stochastically simulating a large subset of all possible combinations of inputs, and then empirically comparing their results to find a set of quasioptimal outcomes. This allows the combinations of inputs to be ranked against each other without the need to perform differential algebra on a fitness function. Example techniques include Genetic Algorithms, Simulated Annealing, Particle Swarm Optimisation, and Ant Colony Optimisations. In many cases this is achieved without even the need for a single all-encompassing fitness function describing the relationships between all inputs. Different variants of the metaheuristic Multi-Objective Optimisation algorithms have their own properties, benefits and drawbacks [9]. For example, the identification of ranking and mutation schemes in Genetic Algorithms is a challenging application-specific making Genetic Algorithms inappropriate for many problems. However, other biologically-inspired algorithms, e.g. Simulated Annealing, Particle Swarm Optimisation, Ant Colony Optimisations etc., are becoming increasing popular $[9][10][11][13]$. In their own way each is focused on the efficient and satisfactory of finding quasi-optimal solutions to problems that cannot be solved numerically. Here we assert that metaheuristic MOO techniques provide practical potential for some of the problems in policy decomposition and refinement.

\section{Methodology}

This research aims to demonstrate the applicability of metaheuristic Multi-Objective Optimisation algorithms in the field of policy decomposition for autonomic governance. This research focuses on how a subjective and vague goal concept can be decomposed into a set of more concrete and measurable concepts, many of which will be interdependent and conflicting. Once decomposed into a set of more manageable concepts, lower level domain specific functions will be available to describe parts of these sub-interrelationships between some of the objectives. In many cases there will not exist a single function to describe how they together contribute to the goal concept. Even if such a function did exist, it would in many cases be too difficult to solve for global optimality across all of the constituent concepts.

Once the constituent conflicting manageable concepts which contribute to the overriding governance concept have each been described in terms of how they are constrained against only a small subset of the other conflicting concepts, each sub-group of related subobjectives can be optimised amongst themselves using a metaheuristic simulation. Assuming these constraint sets contain a degree of overlap for interdependent constraints, the results of each of these individual optimisations can then be combined and interpolated to form a single set of balancing optimal values for all of the conflicting subobjective. Assuming N different constituent concepts, this reduces the problem from an $\mathrm{N}-$ Order differential problem, to a set of smaller-order or single-order problems, none of which will require differentiation. So instead of solving a $\mathrm{N}$-Order differential equation, should one exist, the collection of smaller order problems are simulated and combined, resulting in an N-Dimensional lookup table of balancing levels for the set of constituent sub-concepts that will optimise the original goal concept.

As a concrete example, consider an autonomic service for mobile devices that coordinates information access from a 3rd party telecoms network. Many aspects of the management of the service will be derived from a service 
level agreement (SLA) between the service provider and the network provider. A high-level governance directive may specify that a high quality video streaming service should be enabled in the system, yet maintain Quality of Service (QoS) constraints. To simplify this example, we will assume that QoS is determined by restricted but sufficient network connectivity, minimised battery usage, restricted screen size and restricted memory usage (of course there will be many more contributing factors). However, these factors conflict in a number of ways. Increased bandwidth required for video streaming will make demands on the network connectivity. Networking capabilities can be improved by caching. Caching will make demands on memory usage. In addition video rendering will make demands on display resources, battery usage and CPU usage. Rather than trying to form a single all-encompassing equation for mobile device resource usage in order that it can be globally optimised using differential algebra, simpler functions are available to link many of the sub-concepts. For example, the display, memory, battery and CPU usage can be derived from video type and quality. Caching requirements are based on network availability and video characteristics.

By stochastically simulating and optimising the effects of some or all of these interrelationships using Genetic Algorithms, or similar MOO algorithms, a set of results similar to the following figures would emerge. The first two diagrams (fig. 1) show a reduced sample set of simulated outcomes when trying to balance two sets of conflicting inputs. Here the origin in the bottom left of each graph would be an ideal (but impossible) outcome. The (reduced) set of point solutions are compared against each other to find which sets of solutions "dominate" each other, i.e. are superior along all axes or criteria. As the simulation runs, the "non-dominated" set of solutions that dominate all other solutions, but not each other, approximate the "Pareto non-dominated front" [9][11][12] as shown in fig. 1 .

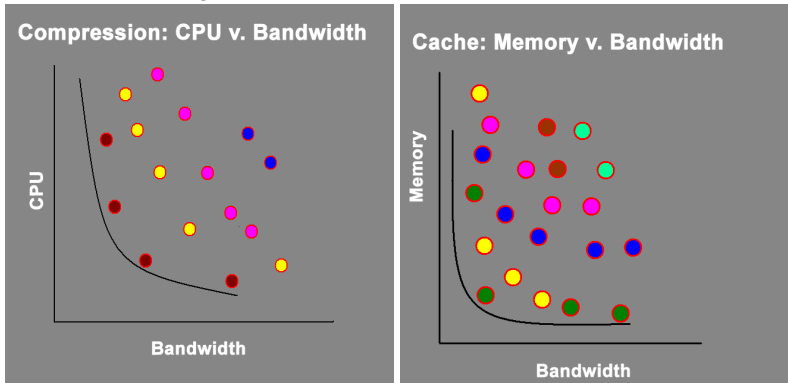

Fig 1: Set of non-dominated data-point solutions for 2 simulation outcomes

The second two sample graphs (fig.2) show plots of convex Pareto-optimal solutions of the set of quasi-optimal combinations of those inputs (Pareto non-dominated front) formed into a curve using non-linear regression techniques.
The Pareto front, which is usually convex, describes the most optimal trade off of alternative combinations of inputs, depending on the relative priority of one input over the other.
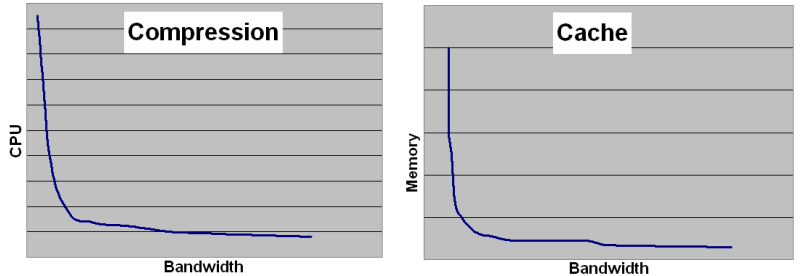

Fig 2: Pareto fronts for the 2 simulation outcomes (Derived from fig. 1)

These Pareto-optimal data-sets for conflicting inputs can be combined using statistical regression techniques into an $\mathrm{N}$-Dimensional dataset by interpolation. The diagram below (fig. 3) shows a sample 3-Dimesional Paretooptimal front that can be generated from three or less sets 2-D Pareto-optimal front plots. Here each dimension represents one of the conflicting inputs.

Due to the overlapping of these constraints, and in this case the use of common resources, these trade-offs can be combined. Each of these should be optimised yet be constrained against each other to maintain the QoS guarantees for not just the managed video service but also for the other services that depend on the information delivery service. Based on the set of trade-offs that are possible, and the optimality of each of these trade-offs, the Governor goals to weight towards one objective or another can be easily achieved. From the 3D plot below, it can be seen that the most optimal solution could be found by calculating the minimum distance from the Origin (ideal but impossible solution) to the surface of the graph, by making use of the standard Euclidean distance calculation,

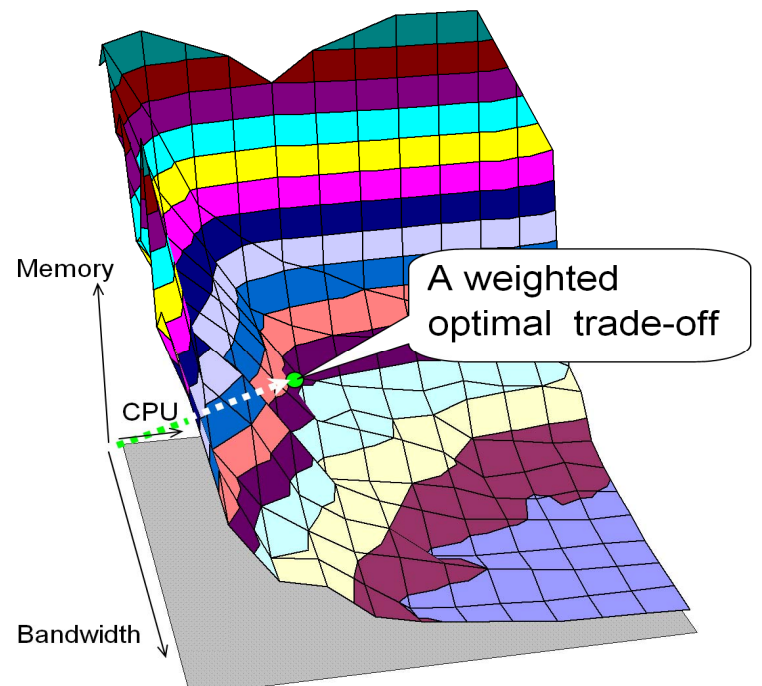

Fig 3: Visualisation of the dataset found by combining and interpolating the results of individual simulation 
or perhaps another Minkowski distance calculation. This assumes that each of the constraint objectives has an equal priority or weighting. If however, one or more of the subgoals that make up the governance goal was more important than others, weightings could be applied to particular dimensions, and the weighted optimum solution found by calculating the intersection of the surface with a weighted vector originating at the Origin. This priority of some objectives over others would typically be dictated by the Governor that oversees the operation of the autonomic system.

A key point to note at this stage is that the MOO simulation does not need to be performed each time a policy is used. The simulations only need to run once, producing a look-up table, and it is this lookup table that is queried in an efficient manner when the policy is being used. The weighting of constraints and the dynamic lookup of a resulting globally optimum balance of constraints would be a lightweight operation and so could be performed dynamically as the Governor's priorities shift. It is only when the underlying constraints that characterise the managed system change that the look-up table needs to be repopulated and it is only the individual simulations that refer to or overlap with that changed characteristic that need to be rerun.

\section{Justification}

Heuristic Multi-Objective Optimisation (MOO) techniques have been used to solve a large number of diverse problems where the conflicting constraints must be optimised to produce a quasi-optimum combined outcome. This is particularly the case where the generation of a single all-encompassing utility function is impractical or cannot be solved for optimality. Due to the stochastic heuristic nature of MOO techniques, many of the algorithms require substantial amounts of time and resources to find a complete and satisfactory solution for the problem. However, much of the recent research in this area has focussed on tweaking or directing the operation of the algorithms to avoid wasted computation based on obviously sub-optimal solutions. In parallel with this research the cheap availability of ever more powerful computers make this problem solving paradigm a practical possibility for problems that have not been solved.

In the domain of autonomic policy decomposition, by definition, the goals that a governor would provide to constrain the operation of an autonomic system would be subjective in nature, since the directive will not be based on what the governor knows about what the system can do, rather than how the system operates. How the system actually adapts will be clearly understood at a lower level of abstraction by the system provider, with management of those adaptation abstracted for the Governor. Any complex system will have domain experts at the "coalface", who are very familiar with how the constituent parts of the system can be managed, and particularly aware of the operating constraints of those constituent parts. In the unlikely event that the governance goals map directly to lower-level management policies, then the decomposition of that governance is trivial. The much more likely event, especially in a complex composed system, is that the governance goals can only be achieved by balancing conflicting and constrained operational goals. This problem forms the key obstacle to general-purpose policy-decomposition.

\section{Conclusion}

Leading experts have identified policy refinement as the primary critical issue in policy based management, where policy-based management is one of the key requirements to achieve the vision of autonomic management [14]. This combination of Multi-Objective Optimisation has not been previously applied in the domain of administering the governance of managed systems, and particularly not in the domain of policy engineering for autonomic systems. This research is innovative in terms of investigating how service management goals can be decomposed into low level configurations and metrics suitable for adapting and reconfiguring services.

However, how such a mechanism would be actually used to manage a system is another debate. As yet many users would be uncomfortable with a fully automated heuristic approach to policy decomposition and so would demand validation of the results of the decomposition and refinement before applying the results. This approach could therefore initially act as a mixed initiative support tool for policy design. Over time, as trust in the system grows, the evolving governance dynamic of the management system would allow existing constraints to be modified or new constraints to be merged, thus resulting in a truly evolving autonomic management system.

\section{References}

[1] Kephart, J., Chess D., "The vision of autonomic computing,” IEEE Computer, 36(1): 41-50, 2003

[2] Lupu, E., Sloman, M., "Conflicts in Policy-Based Distributed Systems Management", IEEE Transactions on software engineering, 25(6):852-869, 1999

[3] Bandara, A., Lupu, E., Moffett, J., Russo, A., "A Goalbased Approach to Policy Refinement", Workshop on Policies for Distributed Systems and Networks (POLICY'04), New York, NY, 7-9 June 2004

[4] Sloman, M., "Policy Driven Management for Distributed Systems", Journal of Network and Systems Management, 2(4):333-360, 1994

[5] Moffet, J., Sloman, M., "Policy Hierarchies for Distributed Systems Management," IEEE Journal on Selected Areas in Communications, Special Issue on Network Management, 11(9):1404-1414, 1993 
[6] Beigi, M., Calo, S., Verma, D., "Policy Transformation Techniques in Policy-based Systems Management", Workshop on Policies for Distributed Systems and Networks (POLICY'04), New York, NY, 7-9 June 2004

[7] Rubio-Loyola, J., Serrat, J., Charalambides, M., Flegkas, P., Pavlou, G., "A methodological approach toward the refinement problem in policy-based management systems", IEEE Communications, 44(10):60-68, 2006

[8] Keeney, J., Carey, K., Lewis, D., O'Sullivan, D., Wade, V., "Ontology-based Semantics for Composable Autonomic Elements", Workshop on AI in Autonomic Communications at the 19th International Joint Conference on Artificial Intelligence (IJCAI), Edinburgh, Scotland. 30 Jul 2005

[9] Kalyanmoy Deb, "Multi-Objective Optimization Using Evolutionary Algorithms" John Wiley \& Sons, 2001, ISBN: 047187339X

[10] Carlos A. Coello Coello, "An Introduction to Evolutionary Algorithms and Their Applications". Proceedings of the 5th International School and
Symposium on Advanced Distributed Systems: (ISSADS 2005), Guadalajara, Mexico, January 24-28, 2005.

[11] Eckart Zitzler and Lothar Thiele, "Multiobjective Evolutionary Algorithms: A Comparative Case Study and the Strength Pareto Approach", IEEE Transactions on Evolutionary Computation, 3(4): 257-271, 1999

[12] Van Veldhuizen, D.A., Lamont, G.B.: "Evolutionary computation and convergence to a Pareto front". In: Koza, J.R., et al. (eds.) Genetic Programming Conference, Late Breaking Papers, pp. 221-228, 1998

[13] Coello Coello, C., A., "Repository on Evolutionary Multiobjective Optimization", 2005, [Online] Available: http://delta.cs.cinvestav.mx/ ccoello/EMOO/ [Accessed: March 6, 2008]

[14] "A Roadmap to Policy Research: Accomplishments and Future Challenges", Expert panel discussion at Workshop on Policies for Distributed Systems and Networks (POLICY'05), Stockholm, Sweden, 6-8 June 2005 